\title{
Giant enhancement in Rashba spin-Seebeck effect in NiFe/p-Si thin films
}

\author{
Ravindra G. Bhardwaj ${ }^{1}$, Paul C Lou ${ }^{1}$ and Sandeep Kumar ${ }^{1,2^{*}}$ \\ ${ }^{1}$ Department of Mechanical Engineering, University of California, Riverside, CA 92521, USA \\ ${ }^{2}$ Materials Science and Engineering Program, University of California, Riverside, CA 92521, \\ USA
}


Abstract

The spin-Seebeck effect mediated thermoelectric energy conversion can provide efficient alternative to traditional thermoelectrics for waste heat recovery. To achieve this goal, efficient spin to charge conversion using earth-abundant materials is essential. Proximity induced Rashba effect arises from the charge potential mediated by structural inversion asymmetry, which has been reported in Si thin films and can be manipulated by controlling the thickness of Rashba layer. We demonstrate a giant Rashba spin-Seebeck effect in NiFe/p-Si (polycrystalline) bilayer thin films. The bilayer thin film specimens have p-Si layer thickness of $5 \mathrm{~nm}, 25 \mathrm{~nm}$ and $100 \mathrm{~nm}$ while keeping the NiFe layer thickness at $25 \mathrm{~nm}$. The Rashba spin Seebeck coefficient has been

estimated to be $0.266 \frac{\mu V}{K}$ for $100 \mathrm{~nm} \mathrm{p}$-Si, and increases by an order of magnitude to $2.11 \frac{\mu V}{K}$ for $5 \mathrm{~nm} \mathrm{p}$-Si. The measured spin-Seebeck coefficient in $5 \mathrm{~nm} \mathrm{p}$-Si specimen is one of the largest coefficient ever reported. The measured voltage of $100.3 \mu \mathrm{V}$ is one of the largest reported spinSeebeck voltage, with smallest area of $\sim 160 \times 10 \mu \mathrm{m}^{2}$ used in any spin-Seebeck measurement. This scientific and technological breakthrough using earth abundant elements brings the spin mediated thermoelectric energy conversion for waste heat recovery closer to reality. 
Since the discovery of spin-Seebeck effect (SSE) by Uchida et al. ${ }^{[1]}$, the spin mediated thermoelectric energy conversion has been extensively investigated for ferromagnetic metals, ferromagnetic insulators, antiferromagnetic materials and oxides ${ }^{[2-9]}$. The SSE is an interface effect that generally occurs between a spin polarized ferromagnet(FM) and a normal metal(NM). In SSE, the thermal transport takes place due to the two-step spin dependent process. In the first step, the thermal gradient leads to generation of heat current from the phonon-magnon or phonon-electron interactions ${ }^{[10-11]}$. The heat current leads to generation of spin currents in the spin-polarized material ${ }^{[12]}$. Spin current is in the form of either magnons or spin-polarized current due to electron or combination of both ${ }^{[11]}$. In the second step, spin current is injected across the interface from FM into NM due the spin potential gradient between FM and NM at the interface. The spin to charge conversion takes place in the NM (usually heavy metal) due to inverse spinHall effect. The FM is spin source and the NM is the spin $\operatorname{sink}^{[3]}$. Thus, the advantage of SSE compared to conventional thermoelectric effect is that it uses the properties of two or more materials that can be independently optimized ${ }^{[13]}$. The spin Seebeck effect and inverse spin-Hall effect (ISHE) produce an electric field given by,

$E_{I S H E}=-S \sigma \times \nabla T$

where, $\mathrm{S}$ is spin Seebeck coefficient and $\sigma$ is the spin polarization vector. Since the above equation is similar to equation of anomalous Nernst effect (ANE) voltage (where $\sigma$ is replaced by $M$ (magnetization)) and thus both SSE and ANE have identical symmetry. One of the controlling factors in spin mediated thermoelectric energy conversion is spin to charge conversion due to spin-orbit coupling in NM. The spin Hall Angle (SHA) is the measure of efficiency in conversion of charge current to spin current given by ratio of generated charge current to the injected spin current ${ }^{[14]}$ and vice versa. Pt is the primary material for spin to charge 
conversion due to its large SHA, which can be enhanced by defects and impurities. Extensive research has been reported in methods to enhance the spin-Hall angle for inverse spin Hall effect. These methods include alloying ${ }^{[15]}$ and metastable phases ${ }^{[16-17]}$.

Recently, Bhardwaj et. al. ${ }^{[18]}$ reported SSE and thermal spin galvanic effect (SGE) in $\mathrm{Ni}_{80} \mathrm{Fe}_{20}$ (poly) bilayer specimen without any heavy metal detector. They propose that the spin to charge conversion in $\mathrm{p}-\mathrm{Si}$ layer in the bilayer specimen is due to structure inversion asymmetry of sandwich structure and proximity effect. They observed that the spin-Seebeck coefficient in the bilayer is of the same order as Pt. However, larger values of spin Seebeck Coefficient $\left(S_{L S S E}\right)$ is required to make the efficient spin mediated thermoelectric technologies into reality. The Rashba spin-orbit coupling (SOC) relies on the charge potential due to structure inversion asymmetry (SIA), which can be controlled by reducing the thickness of the sandwiched layer. The Rashba effect mediated spin-Hall magnetoresistance has also been reported in $\mathrm{Ni}_{81} \mathrm{Fe}_{19} / \mathrm{MgO} / \mathrm{p}-\mathrm{Si}^{[19-21]}$ and $\mathrm{Ni}_{81} \mathrm{Fe}_{19} / \mathrm{MgO} / \mathrm{n}-\mathrm{Si}^{[22]}$ thin films as well. The spin-Hall magnetoresistance arises due to ISHE, which is essential for SSE. We hypothesize the reduction in thickness of $\mathrm{p}-\mathrm{Si}$ will increase Rashba SOC, leading to efficient spin to charge conversion. To explore the thickness dependent SSE behavior, we fabricated three specimen having p-Si layer thickness of $5 \mathrm{~nm}, 25 \mathrm{~nm}$ and $100 \mathrm{~nm}$ while keeping the thickness of NiFe at $25 \mathrm{~nm}$. In this work, we demonstrate giant enhancement in spin mediated thermoelectric energy conversion due to efficient spin to charge conversion from Rashba SOC.

The SSE is usually characterized using two universal device configurations- longitudinal spin Seebeck effect (LSSE) and transverse spin Seebeck effect (TSSE) ${ }^{[23]}$. Spin current is parallel to the temperature gradient in $\operatorname{LSSE}^{[24]}$ while it is perpendicular to the temperature gradient in $\operatorname{TSSE}^{[9,25-26]}$. In this work, we use the LSSE configuration to discover the spin mediated 
thermoelectric energy conversion behavior in $\mathrm{NiFe} / \mathrm{p}-\mathrm{Si}$ bilayers as shown in Figure 1 (a). In the LSSE configuration, the temperature gradient across the thin film specimen creates a spin current $\left(J_{s}\right)$, which then get converted into a charge current $\left(J_{c}\right)$ as shown in Figure 1 (a). To fabricate the experimental setup, we take a $\mathrm{Si}$ wafer and deposit $450 \mathrm{~nm}$ of thermal silicon oxide using chemical vapor deposition (CVD). We, then, deposit the NiFe/p-Si (poly) bilayers using the RF sputtering as shown in Figure 1 (b). We deposit three sets of bilayers with $5 \mathrm{~nm}, 25 \mathrm{~nm}$ and 100 $\mathrm{nm}$ of $\mathrm{p}$-Si while keeping the NiFe thickness at $25 \mathrm{~nm}$. We hide three quarters of the wafer and deposit each of the bilayer individually. The p-Si target is B-doped having resistivity of $0.005-$ $0.01 \Omega$-cm. We sputter $50 \mathrm{~nm} \mathrm{MgO}$ to electrically isolate the heater and the specimen. We then deposit heater composed of $\mathrm{Ti}(10 \mathrm{~nm}) / \mathrm{Pt}(100 \mathrm{~nm})$. The insulator and heater deposition are common to all the bilayers devices, which reduces the fabrication induced measurement variations. We acquire experimental data inside a Quantum design Physical property measurement system (PPMS). To ascertain the thermal response characteristics, we acquire the second harmonic response as a function of current at an applied magnetic field of 1500 Oe as shown in Figure 1 (c). We observe that the $V_{2 \omega}$ response shows a relationship having both quadratic and linear terms with respect to the applied heating current. This behavior suggests that the temperature rise across the specimen do not have linear relationship with the square of heating current, which may be due to temperature drop across $\mathrm{MgO}$ (top) and thermal oxide layers (bottom).

(Figure 1)

To investigate the SSE behavior, we acquire the $V_{2 \omega}$ response as a function of magnetic field (1500 Oe to -1500 Oe) applied in the y-direction (normal to the temperature gradient) for all the three specimens. The data is acquired at $10 \mathrm{~K}, 100 \mathrm{~K}$ and $300 \mathrm{~K}$ as shown in Figure 2 a-c. 
The observed behavior demonstrates the magnetic switching behavior for all the thicknesses, which is consistent with SSE and ANE. At $300 \mathrm{~K}$, the $V_{2 \omega}$ for the $5 \mathrm{~nm}, 25 \mathrm{~nm}$ and $100 \mathrm{~nm}$ are $100.3 \mu \mathrm{V}, 36.77 \mu \mathrm{V}$ and $31.08 \mu \mathrm{V}$ respectively. Although the $V_{2 \omega}$ response for the $25 \mathrm{~nm}$ and $100 \mathrm{~nm}$ specimens is similar in magnitude but the magnitude of $V_{2 \omega}$ response for $5 \mathrm{~nm}$ is extremely large. This behavior eliminates ANE as an underlying mechanism for the observed behavior since the NiFe layer thickness is same across all the specimens. For specimen with 5 $\mathrm{nm}$ p-Si layer thickness, the $V_{S S E}$ is $100.3 \mu \mathrm{V}$ at $300 \mathrm{~K}$, which is significantly larger as compared to any other SSE measurement reported in the literature. In addition, the SSE specimen area is $160 \mathrm{X} 10 \mu \mathrm{m}^{2}$ in this study, which is an order of magnitude smaller than the other reported experiments. Notable, this efficient spin mediated thermoelectric energy conversion is achieved without using any heavy metal for spin to charge conversion. This giant enhancement in SSE, presented in this study, is attributed to the proximity induced Rashba SOC in p-Si layer, which increases significantly with reduction in $\mathrm{p}$-Si layer due to structure inversion asymmetry, resulting in the observed behavior. The Rashba SOC may also give rise to spin-galvanic effect (SGE). In the recent work, Bhardwaj et al. ${ }^{[18]}$ reported thermal SGE for an out of plane magnetic field, where its magnitude is reported to be similar to SSE. Here, we undertake a similar experiment to uncover the thermal SGE behavior. We measure the $V_{2 \omega}$ response as a function of magnetic field (from -2500 Oe to $2500 \mathrm{Oe}$ ) applied in the z-direction (parallel to temperature gradient) at $300 \mathrm{~K}$ for a heating current a $20 \mathrm{~mA}$ as shown in the Figure $2 \mathrm{~d}$. The observed $V_{2 \omega}$ response is similar to SSE measurement. The $V_{2 \omega}$ response in case of specimen having $5 \mathrm{~nm} \mathrm{p-Si}$ layer is very large as compared to specimens having $25 \mathrm{~nm}$ and $100 \mathrm{~nm}$ p-Si thick layer. We propose that this out of plane $V_{2 \omega}$ response is due to thermal SGE. 
(Figure 2)

The measurement of $V_{2 \omega}$ response as a function of magnetic field leads to confirmation of the SSE and thermal SGE behavior. To uncover the underlying mechanism of SSE mediated energy conversion behavior, we measure $V_{2 \omega}$ response as a function of temperature from $350 \mathrm{~K}$ to $10 \mathrm{~K}$ under a magnetic field of 1000 Oe applied along the y-direction. We observe a gradual decrease in the $V_{2 \omega}$ response as the temperature is lowered to $10 \mathrm{~K}$ as shown in Figure 3 a. The temperature dependent behavior signifies that the observed $V_{2 \omega}$ response is due to magnon mediated SSE. To further support our argument, we measured angular dependence of the $V_{2 \omega}$ response for a constant applied magnetic field of $2 \mathrm{~T}$ rotated in the yx-plane. For all the specimens, we observe an embeded cosine behavior attributed to SSE. The deviation from the cosine behavior can arise from the thermal SGE contributions.

(Figure 3)

To quantify the SSE in this study, we estimate the longitudinal spin Seebeck coefficient $\operatorname{as}^{[18,27]}$

$S_{L S S E}=\frac{E_{I S H E}}{\nabla T}=\frac{V_{I S H E} t_{F M}}{w_{N M} \Delta T}$

where, $\Delta T$ is the temperature gradient across the sample, $w_{N M}$ is the distance between electrical contact in Normal Metal (NM) $(\sim 160 \mu m), t_{F M}$ is the ferromagnet (FM) material thickness, and $V_{I S H E}$ is the $V_{2 \omega}$ response measured due to ISHE in NM. Using $3 \omega$ method, we can experimentally calculate the temperature gradient between heater and the far field substrate temperature. The temperature gradient between heater and substrate using $3 \omega$ method $^{[28]}$ is given by 
$\Delta T=\frac{4 V_{3 \omega}}{R^{\prime} I_{r m s}}$

Where, $V_{3 \omega}$ is the measured third harmonic response, $R^{\prime}$ is the slope of the resistance as a function of temperature and $I_{r m s}$ is the applied heating current. The heater temperature is estimated to be $\sim 313.7 \mathrm{~K}$ for bilayer specimen having $25 \mathrm{~nm}$ p-Si layer and $313.4 \mathrm{~K}$ in case of $100 \mathrm{~nm}$ p-Si specimen for $R^{\prime}$ of $0.07 \Omega / \mathrm{K}^{[18]}$. We use finite element method (FEM) (COMSOL software) to simulate the temperature gradient across the bilayer specimen, which is essential for spin-Seebeck coefficient. For modeling the temperature gradient, we assumed the $\kappa_{p-S i}$ to be 15 $\mathrm{W} / \mathrm{mK}, 20 \mathrm{~W} / \mathrm{mK}$ and $35 \mathrm{~W} / \mathrm{mK}^{[29-30]}$ for thickness of $5 \mathrm{~nm}, 25 \mathrm{~nm}$ and $100 \mathrm{~nm}$ respectively and $\kappa_{N i F e}$ to be $20 \mathrm{~W} / \mathrm{mK}^{[31]}$. We observe that simulated temperature gradient across the NiFe layer is similar for all the p-Si layers. This observation reinforces that the ANE is not the underlying reason of observed $V_{2 \omega}$ response. Using the simulated temperature gradient across the bilayer, the $S_{L S S E}$ is calculated to be $2.11 \frac{\mu \mathrm{V}}{\mathrm{K}}, 0.506 \frac{\mu \mathrm{V}}{\mathrm{K}}$ and $0.266 \frac{\mu \mathrm{V}}{\mathrm{K}}$ for $5 \mathrm{~nm}, 25 \mathrm{~nm}$ and $100 \mathrm{~nm}$ thick p-Si layer thickness respectively as shown in Figure 3 (c). As stated earlier, the observed SSE behavior is proposed to occur due to proximity Rashba SOC. The Figure 3 (d) shows the mechanism of the observed Rashba SSE behavior. Using ARPES measurement, Gierz et al. ${ }^{[32]}$ demonstrated giant spin-splitting at the $\mathrm{Bi} / \mathrm{Si}$ (111) interface, which is ascribed to structural inversion asymmetry or Rashba effect. This Rashba energy of $140 \mathrm{meV}$ is reported, which is larger than any other semiconductor heterostructure. In addition, anisotropic electronic band structure is reported for Si (110) using ARPES and simulations ${ }^{[33]}$. These measurements support our hypothesis. This study includes a ferromagnet having large spin-orbit coupling ${ }^{[34-35]}$, which will introduce in-plane Rashba spin spitting in addition to lifting the degeneracy of band structure due to ferromagnetic proximity effect as show in Figure 3 (d). While Rashba SOC can 
give rise to giant spin to charge conversion, it may not give rise to ISHE essential for SSE observe in this study. But, Rashba SOC can lift the degeneracy of electronic band structure. The splitting of band structure due to Rashba SOC and ferromagnetic proximity effect can give rise to ISHE. We propose that a combined effect Rashba SOC and ferromagnetic proximity effect leads to the observed giant SSE response in this study. Since, we observe SSE in p-Si layer having thickness of $100 \mathrm{~nm}$, the resulting behavior cannot arise from the two-dimensional electron gas only. The proposed Rashba SOC is bulk, which is consistent with recent reports on $\mathrm{Si}^{[19,22,36]}$. With the reduction in $\mathrm{p}$-Si layer thickness, the Rashba SOC will increase and in turn SSE response, which is supported by our measurements.

The thickness dependent LSSE measurement can be used to calculate the spin-Hall angle and spin diffusion length. Qu et al. ${ }^{[37]}$ used the LSSE measurement to uncover the spin-Hall angles using the following equation-

$\Delta V_{t h}$ or $V_{S S E}=[2 C L \Delta T]\left[\rho(t) \theta_{S H}\right]\left[\frac{\lambda_{S F}}{t} \tanh \left(\frac{t}{2 \lambda_{S F}}\right)\right]$

Where $\Delta V_{t h}$ is the thermal voltage due to SSE, the first factor on the right-hand side [CLAT] relates to spin injection efficiency, the length of the wire, and temperature gradient respectively. The second factor on the right-hand side $\left[\rho(t) \theta_{S H}\right]$ material specific quantity and relates to the spin conductivity. The last factor relates to the spin diffusion length $\left(\lambda_{S F}\right)$ and thickness $(t)$. Note that the equation 4 assumes that the intrinsic spin diffusion length and spin-Hall angle are independent of material thickness. Although this assumption may be true for the intrinsic spin orbit coupling in case of $5 \mathrm{~d}$ heavy metals ${ }^{[15,37]}$ but the Rashba SOC that is responsible for the ISHE is thickness dependent. Hence, the equation 4 cannot quantify the spin transport behavior in $\mathrm{NiFe} / \mathrm{p}-\mathrm{Si}$ bilayer thin films presented in this work. 
Instead of quantitative analysis, we undertake a comparative study of the observed SSE in $\mathrm{NiFe} / \mathrm{p}$-Si bilayer specimen. We analyzed the reported LSSE measurement over the years for various materials. To demonstrate the quantum of spin-Seebeck behavior observed in the present study, we list the reported $\mathrm{V}_{\mathrm{SSE}}>10 \mu \mathrm{V}$ in Table 1, where three articles report spin-Seebeck voltage of more than $100 \mu \mathrm{V}^{[38]}{ }^{[39]}$. Jiang et al. reported a spin-Seebeck voltage of $175 \mu \mathrm{V}$ in Bi doped topological insulator $\left(\mathrm{Sb}_{2} \mathrm{Te}_{3}\right)$, which is probably the highest $\mathrm{V}_{\mathrm{SSE}}$ reported in the literature. While the $\mathrm{V}_{\mathrm{SSE}}$ reported in present study is smaller, but the area of the specimen in present study is $\sim 56.25$ times smaller as well. In addition, the heating power used to achieve the $175 \mu \mathrm{V}$ is $500 \mathrm{~mW}$ whereas the heating power of $17.6 \mathrm{~mW}$ is used in this study to generate 100.3 $\mu \mathrm{V}$. This observation clearly demonstrates the superiority of the thermoelectric efficiency in the $\mathrm{NiFe} / \mathrm{p}-\mathrm{Si}$ bilayer system. Lin et al. ${ }^{[40]}$ demonstrated a similar $\mathrm{V}_{\text {SSE }}$ with $\mathrm{NiO}$ spacer in between YIG and Pt. They also report the highest SSC of $6 \mu \mathrm{V} / \mathrm{K}$. The SSC is proportional to the thickness of the FM layer (YIG), which is $500 \mu \mathrm{m}$ in this reported study. In addition, the specimen area is three orders of magnitude larger as compared to present study. Ramos et al. demonstrated a giant spin-Seebeck voltage in $\mathrm{Fe}_{3} \mathrm{O}_{4} / \mathrm{Pt}$ system using a spin-Hall thermopile setup. But the specimen area is 3 orders of magnitude larger than the specimen area in this study. In addition, the spin-Hall thermopile configuration can be applied to NiFe/p-Si bilayer system as well to achieve even higher voltages. We have listed other reports of large spin-Seebeck voltage. All specimens in the listed studies consist of areas that are three orders of magnitude larger, with spin-Seebeck voltages that are an order of magnitude smaller than presented in this work.

Table 1. The summary of largest spin-Seebeck voltages and corresponding spin source, spin detector, specimen dimensions and spin-Seebeck coefficient. 


\begin{tabular}{|c|c|c|c|c|c|c|}
\hline$\Delta \mathbf{V}(\boldsymbol{\mu} \mathbf{V})$ & $\begin{array}{c}\text { Spin } \\
\text { source }\end{array}$ & Detector & Specimen $(\mathbf{L} \times \mathbf{B})$ & $\begin{array}{c}\text { SSC } \\
(\mu \mathrm{V} / \mathrm{K})\end{array}$ & $\begin{array}{c}\text { Dimensional } \\
\text { Normalization }\end{array}$ & Ref. \\
\hline$\sim 175$ & YIG & $\left(\mathrm{Bi}_{\mathrm{x}} \mathrm{Sb}_{1-\mathrm{x}}\right)_{2} \mathrm{Te}_{3}$ & $900 \mu \mathrm{m} \times 100 \mu \mathrm{m}$ & $\begin{array}{c}\text { Not } \\
\text { reported }\end{array}$ & N/A & {$[38]$} \\
\hline$\sim 175$ & YIG & $\mathrm{Pt} / \mathrm{NiO}$ & $7 \mathrm{~mm} \times 2 \mathrm{~mm}$ & 6 & Yes & {$[40]$} \\
\hline 100.3 & $\mathrm{NiFe}$ & p-Si (poly) & $\begin{array}{c}160 \mu \mathrm{m} \times 10 \mu \mathrm{m} \\
\text { (smallest) }\end{array}$ & $0.2-2.2$ & Yes & $\begin{array}{l}\text { This } \\
\text { study }\end{array}$ \\
\hline$\sim 100$ & $\mathrm{Fe}_{3} \mathrm{O}_{4}$ & $\mathrm{Pt}$ & $\begin{array}{l}\text { Spin-Hall } \\
\text { thermopile }(7 \mathrm{~mm} \times \\
2 \mathrm{~mm})\end{array}$ & $\begin{array}{c}\text { Not } \\
\text { reported }\end{array}$ & N/A & [39] \\
\hline$\sim 26$ & YIG & $\mathrm{Pt}$ & $6 \mathrm{~mm} \times 2 \mathrm{~mm}$ & 0.100 & No & {$[26]$} \\
\hline $\begin{array}{c}\sim 25 \text { and } \\
\sim 12\end{array}$ & $\mathrm{Fe}_{3} \mathrm{O}_{4}$ & $\mathrm{Pt}$ & $7 \mathrm{~mm} \times 2 \mathrm{~mm}$ & $\begin{array}{c}0.03 \text { and } \\
0.7\end{array}$ & Yes & {$[13,41]$} \\
\hline$\sim 18$ & YIG & $\mathrm{Pt}$ & $10 \mathrm{~mm} \times 2.3 \mathrm{~mm}$ & 1.500 & No & {$[42]$} \\
\hline$\sim 12$ & $\mathrm{NiFe}_{2} \mathrm{O}_{4}$ & $\mathrm{Pt}$ & $8 \mathrm{~mm} \times 5 \mathrm{~mm}$ & $\begin{array}{l}0.030 \\
-0.020\end{array}$ & No & {$[43]$} \\
\hline
\end{tabular}

In conclusion, we report a giant increase in $\mathrm{SSE}$ in $\mathrm{NiFe}(25 \mathrm{~nm}) / \mathrm{p}-\mathrm{Si}$ (polycrystalline) bilayer specimens having p-Si thickness of $5 \mathrm{~nm}, 25 \mathrm{~nm}$ and $100 \mathrm{~nm}$. The spin-Seebeck voltage shows a three-fold increase in case of $5 \mathrm{~nm}$ p-Si specimen as compared to the $25 \mathrm{~nm}$ and $100 \mathrm{~nm}$ p-Si specimens. The inverse spin-Hall effect is proposed to occur due to proximity induced Rashba spin orbit coupling at the $\mathrm{NiFe} / \mathrm{p}-\mathrm{Si}$ interface. This observation eliminates the requirement of heavy metal ( $\mathrm{Pt}$ or $\mathrm{Ta}$ ) for spin to charge conversion. The largest spin-Seebeck coefficient reported in this study is a technological breakthrough, which may help in realization of waste heat recovery applications using spin-Seebeck effect.

\section{Acknowledgement}


RGB and PCL has equal contribution to this work.

\section{References}

[1] K. Uchida, S. Takahashi, K. Harii, J. Ieda, W. Koshibae, K. Ando, S. Maekawa, E. Saitoh, Nature 2008, 455, 778.

[2] A. Kirihara, K.-i. Uchida, Y. Kajiwara, M. Ishida, Y. Nakamura, T. Manako, E. Saitoh, S. Yorozu, Nat Mater 2012, 11, 686.

[3] S. R. Boona, R. C. Myers, J. P. Heremans, Energy \& Environmental Science 2014, 7, 885.

[4] A. B. Cahaya, O. A. Tretiakov, G. E. W. Bauer, Appl. Phys. Lett. 2014, 104, 042402.

[5] A. B. Cahaya, O. A. Tretiakov, G. E. W. Bauer, IEEE Trans. Magn. 2015, 51, 1.

[6] Q. Zhiyong, H. Dazhi, K. Takashi, U. Ken-ichi, S. Eiji, Applied Physics Express 2015, 8, 083001.

[7] S. M. Wu, W. Zhang, A. KC, P. Borisov, J. E. Pearson, J. S. Jiang, D. Lederman, Phys. Rev. Lett. 2016, 116, 097204.

[8] P. Sheng, Y. Sakuraba, Y.-C. Lau, S. Takahashi, S. Mitani, M. Hayashi, Science Advances 2017, 3.

[9] K. Uchida, J. Xiao, H. Adachi, J. Ohe, S. Takahashi, J. Ieda, T. Ota, Y. Kajiwara, H. Umezawa, H. Kawai, G. E. W. Bauer, S. Maekawa, E. Saitoh, Nat Mater 2010, 9, 894.

[10] H. Adachi, K.-i. Uchida, E. Saitoh, J.-i. Ohe, S. Takahashi, S. Maekawa, Appl. Phys. Lett. $2010,97$.

[11] J. Xiao, G. E. W. Bauer, K.-c. Uchida, E. Saitoh, S. Maekawa, Phys. Rev. B 2010, 81, 214418.

[12] K. Uchida, T. Ota, H. Adachi, J. Xiao, T. Nonaka, Y. Kajiwara, G. E. W. Bauer, S. Maekawa, E. Saitoh, J. Appl. Phys. 2012, 111, 103903.

[13] R. Ramos, T. Kikkawa, K. Uchida, H. Adachi, I. Lucas, M. H. Aguirre, P. Algarabel, L. Morellon, S. Maekawa, E. Saitoh, M. R. Ibarra, Appl. Phys. Lett. 2013, 102, 072413.

[14] L. Wang, R. J. H. Wesselink, Y. Liu, Z. Yuan, K. Xia, P. J. Kelly, Phys. Rev. Lett. 2016, 116, 196602.

[15] D. Qu, S. Y. Huang, G. Y. Guo, C. L. Chien, Phys. Rev. B 2018, 97, 024402.

[16] L. Liu, C.-F. Pai, Y. Li, H. W. Tseng, D. C. Ralph, R. A. Buhrman, Science (New York, N.Y.) 2012, 336, 555.

[17] Q. Hao, W. Chen, G. Xiao, Appl. Phys. Lett. 2015, 106, 182403.

[18] R. G. Bhardwaj, P. C. Lou, S. Kumar, Appl. Phys. Lett. 2018, 112, 042404.

[19] P. C. Lou, S. Kumar, physica status solidi (b) 2017, DOI: 10.1002/pssb.2017005451700545.

[20] P. C. Lou, S. Kumar, Solid State Commun. 2017, 259, 24.

[21] P. C. Lou, W. P. Beyermann, S. Kumar, J. Appl. Phys. 2017, 122, 123905.

[22] P. C. Lou, S. Kumar, Journal of Magnetism and Magnetic Materials 2018, 452, 129.

[23] M. Weiler, M. Althammer, F. D. Czeschka, H. Huebl, M. S. Wagner, M. Opel, Phys. Rev. Lett. 2012, 108, 106602.

[24] K. Uchida, M. Ishida, T. Kikkawa, A. Kirihara, T. Murakami, E. Saitoh, Journal of Physics: Condensed Matter 2014, 26, 343202.

[25] A. Sola, M. Kuepferling, V. Basso, M. Pasquale, T. Kikkawa, K. Uchida, J. Appl. Phys. 2015, 117, 17C510. 
[26] K.-i. Uchida, T. Nonaka, T. Ota, E. Saitoh, Appl. Phys. Lett. 2010, 97, 262504.

[27] A. Sola, P. Bougiatioti, M. Kuepferling, D. Meier, G. Reiss, M. Pasquale, T. Kuschel, V. Basso, Scientific Reports 2017, 7.

[28] D. G. Cahill, Rev. Sci. Instrum. 1990, 61, 802.

[29] P. E. Hopkins, L. M. Phinney, J. Heat Transfer 2009, 131, 043201.

[30] W. Liu, M. Asheghi, J. Appl. Phys. 2005, 98, 123523.

[31] A. D. Avery, S. J. Mason, D. Bassett, D. Wesenberg, B. L. Zink, Phys. Rev. B 2015, 92, 214410.

[32] I. Gierz, T. Suzuki, E. Frantzeskakis, S. Pons, S. Ostanin, A. Ernst, J. Henk, M. Grioni, K. Kern, C. R. Ast, Phys. Rev. Lett. 2009, 103, 046803.

[33] S. Y. Matsushita, A. Takayama, E. Kawamoto, C. Hu, S. Hagiwara, K. Watanabe, T. Takahashi, S. Suto, Phys. Rev. B 2017, 96, 125302.

[34] B. F. Miao, S. Y. Huang, D. Qu, C. L. Chien, Phys. Rev. Lett. 2013, 111, 066602.

[35] A. Hrabec, F. J. T. Gonçalves, C. S. Spencer, E. Arenholz, A. T. N'Diaye, R. L. Stamps, C. H. Marrows, Phys. Rev. B 2016, 93, 014432.

[36] P. C. Lou, S. Kumar, Journal of Physics: Condensed Matter 2018, 30, 145801.

[37] D. Qu, S. Y. Huang, B. F. Miao, S. X. Huang, C. L. Chien, Phys. Rev. B 2014, 89, 140407.

[38] Z. Jiang, C.-Z. Chang, M. R. Masir, C. Tang, Y. Xu, J. S. Moodera, A. H. MacDonald, J. Shi, Nat Commun 2016, 7, 11458.

[39] R. Ramos, A. Anadón, I. Lucas, K. Uchida, P. A. Algarabel, L. Morellón, M. H. Aguirre, E. Saitoh, M. R. Ibarra, APL Materials 2016, 4, 104802.

[40] W. Lin, K. Chen, S. Zhang, C. L. Chien, Phys. Rev. Lett. 2016, 116, 186601.

[41] R. Ramos, T. Kikkawa, A. Anadón, I. Lucas, K. Uchida, P. A. Algarabel, L. Morellón, M. H. Aguirre, E. Saitoh, M. R. Ibarra, AIP Advances 2017, 7, 055915.

[42] S. M. Rezende, R. L. Rodríguez-Suárez, R. O. Cunha, A. R. Rodrigues, F. L. A. Machado, G. A. Fonseca Guerra, J. C. Lopez Ortiz, A. Azevedo, Phys. Rev. B 2014, 89, 014416.

[43] D. Meier, T. Kuschel, L. Shen, A. Gupta, T. Kikkawa, K. Uchida, E. Saitoh, J. M. Schmalhorst, G. Reiss, Phys. Rev. B 2013, 87, 054421. 


\section{List of Figures-}

Figure 1. (a) the schematic showing the longitudinal spin-Seebeck effect measurement setup, (b) the scanning electron micrograph showing the experimental setup, and (c) the second harmonic response as a function of heating current for an applied magnetic field of 1500 Oe.

Figure 2. The longitudinal spin-Seebeck effect measurement for magnetic field applied along the y-direction at $300 \mathrm{~K}, 100 \mathrm{~K}$ and $10 \mathrm{~K}$ for the specimen having $25 \mathrm{~nm}$ of NiFe layer and $\mathrm{p}-\mathrm{Si}$ layer thickness of (a) $100 \mathrm{~nm}$, (b) $25 \mathrm{~nm}$ and (c) $5 \mathrm{~nm}$, and (d) the thermal spin galvanic effect measurement at $300 \mathrm{~K}$ for field applied along z-direction for specimen having $\mathrm{p}$-Si layer thickness of $100 \mathrm{~nm}, 25 \mathrm{~nm}$ and $5 \mathrm{~nm}$ of $\mathrm{p}-\mathrm{Si}$.

Figure 3. The spin-Seebeck voltage response for bilayer specimen having p-Si layer thickness of $100 \mathrm{~nm}, 25 \mathrm{~nm}$ and $5 \mathrm{~nm}$ (a) as a function of temperature from $350 \mathrm{~K}$ to $5 \mathrm{~K}$, (b) angular dependence in the yx-plane for an applied magnetic field of $2 \mathrm{~T}$, (c) the calculated spin-Seebeck coefficient as a function of thickness, and (d) schematic of the proposed mechanism for the observed behavior showing proximity Rashba spin splitting and resulting spin to charge conversion. 


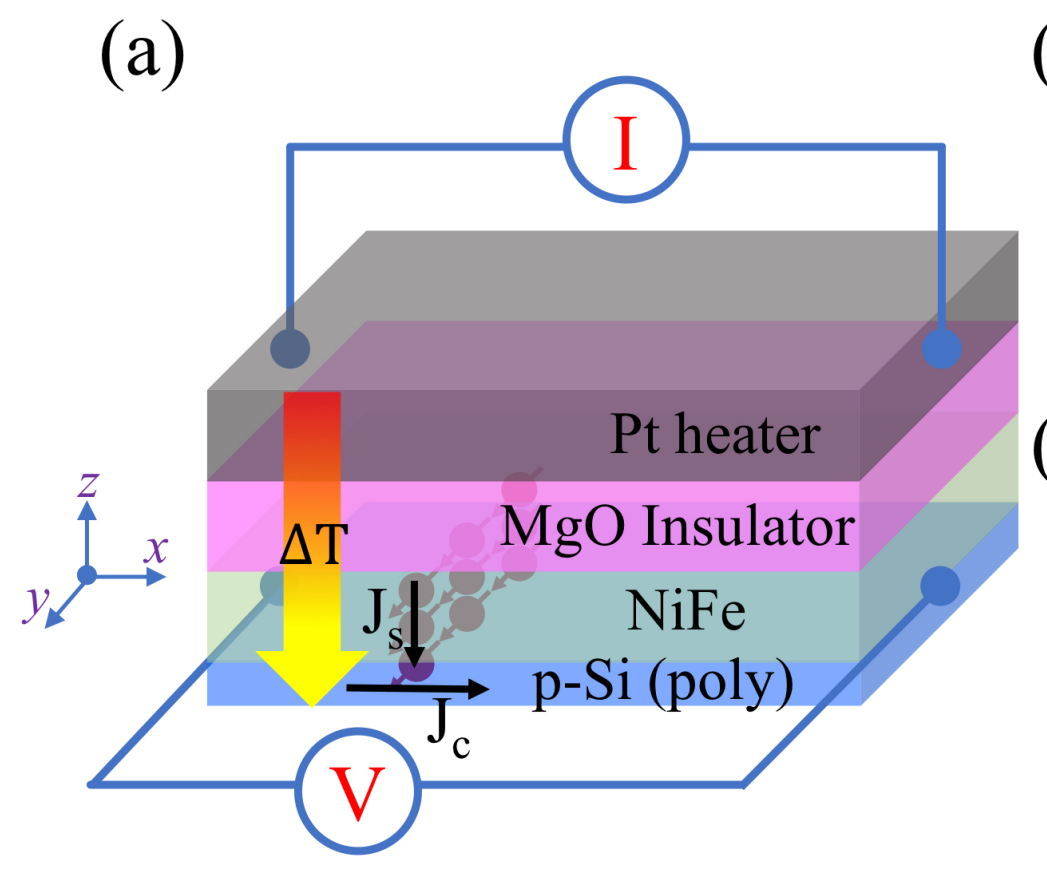

(b)

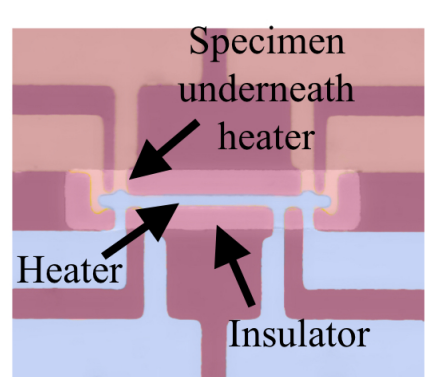

(c)
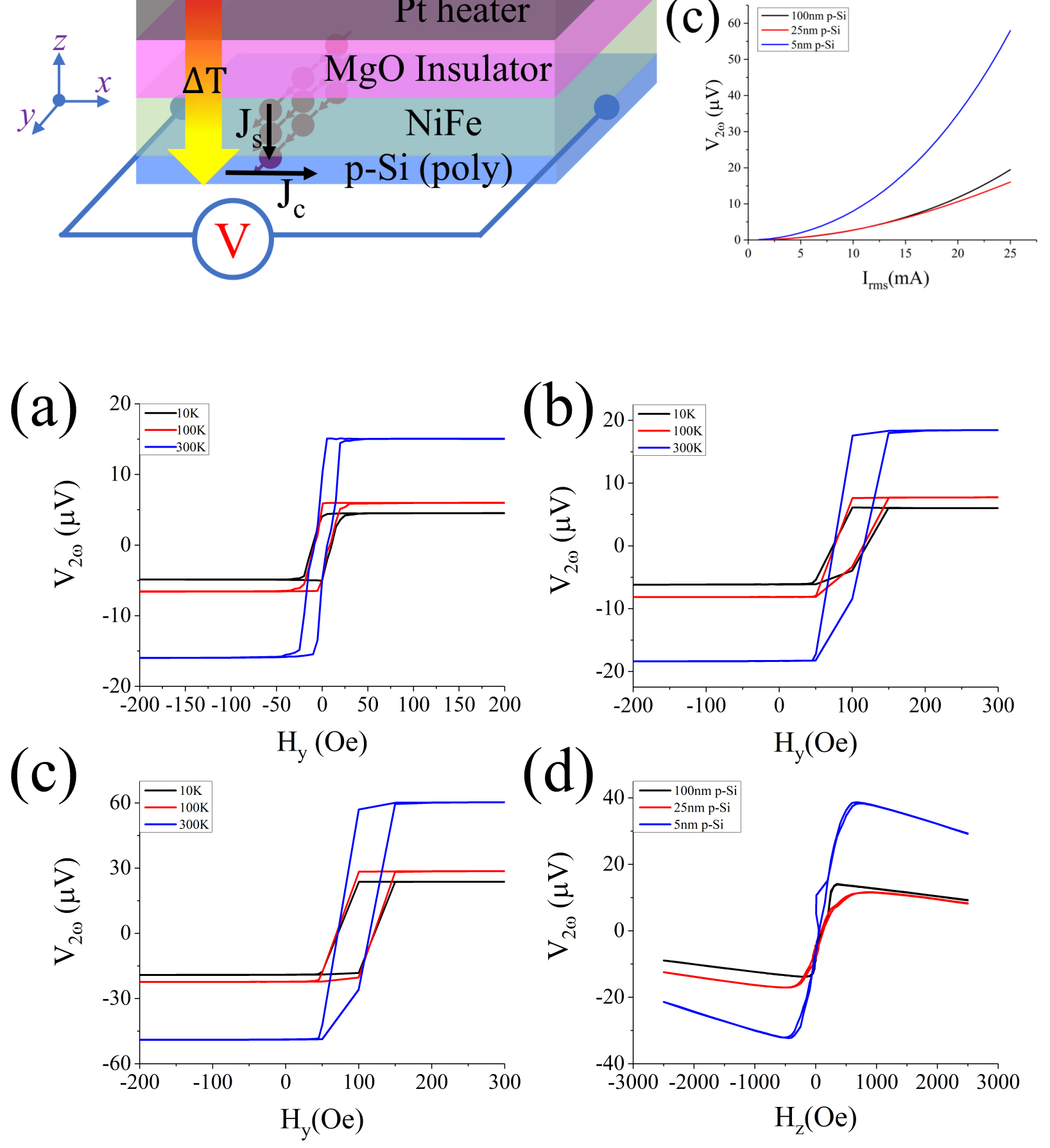

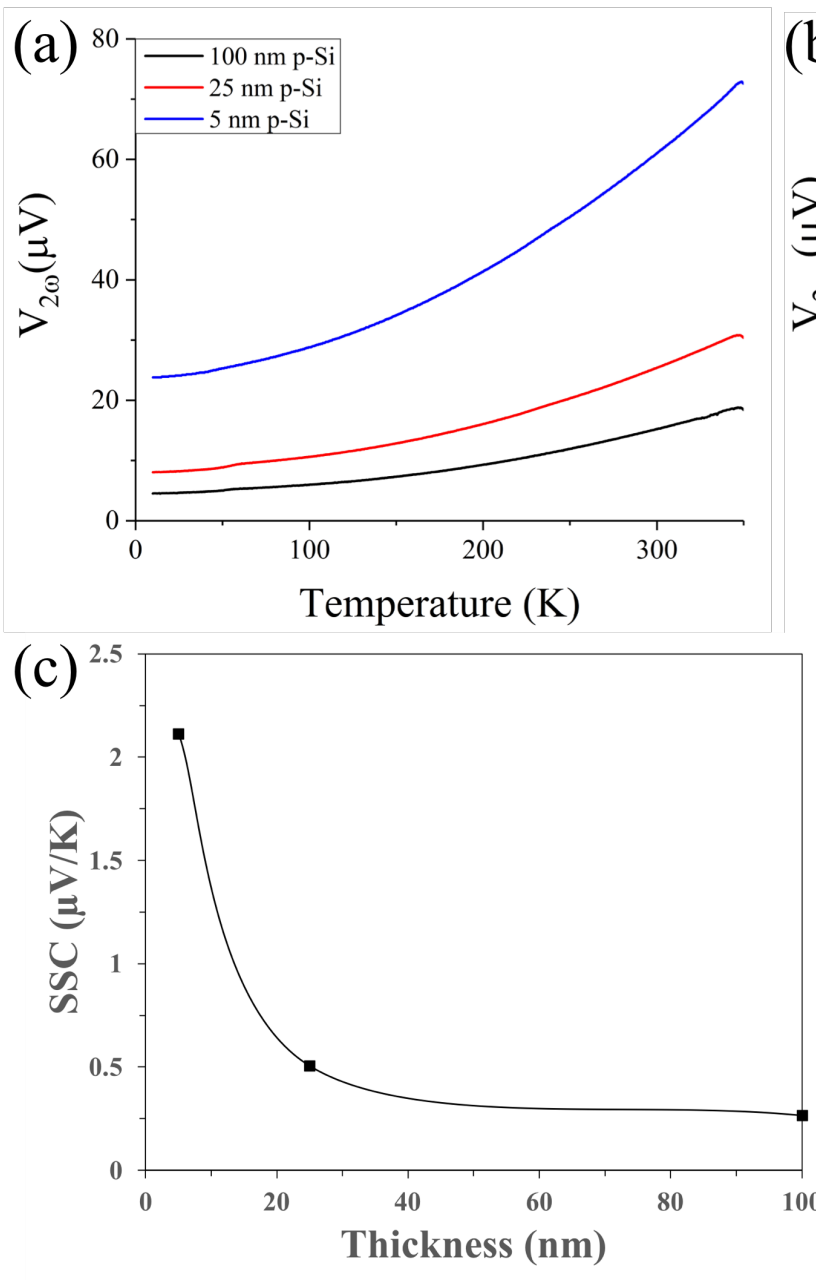

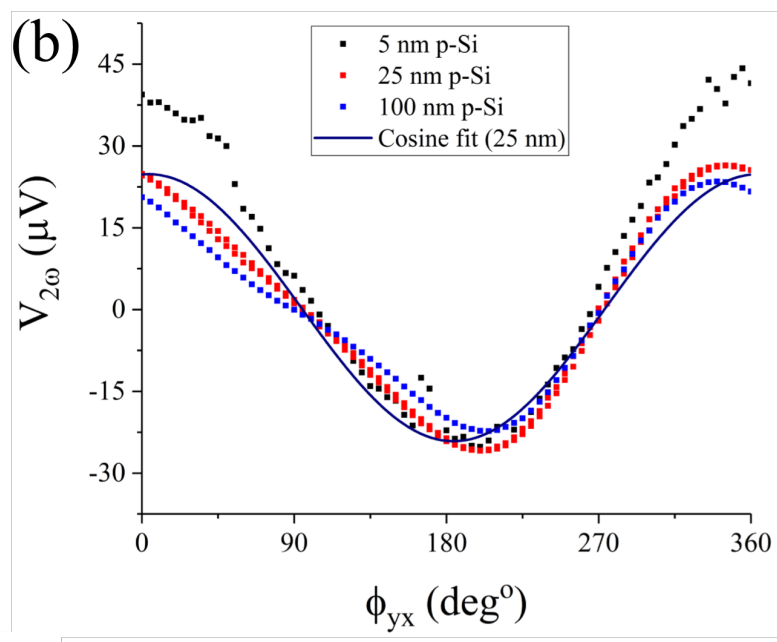

(d)

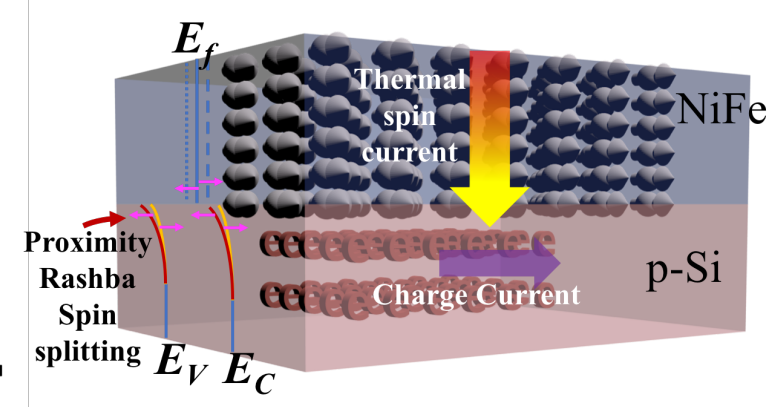

